\title{
Capitalizing on the mathematical foundation of cardiovascular physiology and the image processing capabilities of the human mind
}

\author{
Michael K. Pasque, MD \\ From the Division of Cardiothoracic Surgery, Department of Surgery, Washington University School of Medicine, \\ St Louis, Mo. \\ Disclosures: Author has nothing to disclose with regard to commercial support. \\ Received for publication April 22, 2016; accepted for publication April 26, 2016; available ahead of print May 24, \\ 2016. \\ Address for reprints: Michael K. Pasque, MD, Washington University School of Medicine, 660 S Euclid Ave, \\ Campus Box 8234, St Louis, MO 63110 (E-mail: pasquem@wustl.edu). \\ J Thorac Cardiovasc Surg 2016;152:428-9 \\ $0022-5223 / \$ 36.00$ \\ Copyright (C) 2016 by The American Association for Thoracic Surgery \\ http://dx.doi.org/10.1016/j.jtcvs.2016.04.075
}

\begin{abstract}
Philosophy [i.e. physics] is written in this grand book-I mean the universe-which stands continually open to our gaze, but it cannot be understood unless one first learns to comprehend the language and interpret the characters in which it is written. It is written in the language of mathematics, and its characters are triangles, circles and other geometrical figures, without which it is humanly impossible to understand a single word of it; without these, one is wandering around in a dark labyrinth.
\end{abstract}

\section{-Galileo Galilei ${ }^{1}$}

We cannot help but find ourselves cheering on the efforts in this issue of The Journal of Oechtering and colleagues, ${ }^{2}$ who have combined their extraordinary magnetic resonance imaging visualization, computational, and surgical capabilities to examine the postoperative hemodynamics of an anatomically shaped sinus prosthesis in valve-sparing aortic root replacement. We want to believe that a Dacron graft that closely mimics the natural shape of the aortic root will provide an environment favorable to long-term native leaflet preservation. The methodology used by this group in exploring the hemodynamic consequences of this geometric modification can only be described as elegant. To appreciate this work fully, not only for its impact on valve-sparing root replacement procedures but also for its prophetic use of advanced computational methods to visualize the resulting hemodynamic consequences, the reader is encouraged to examine not only the color images on the printed page but also the stunning online videos.

Although the arduous task of scrutinizing the details of this investigation's statistical analysis might bore most practicing cardiac surgeons, the visual display of their results allows us to do what we, as human beings, do best. We can use our own advanced image-processing software,

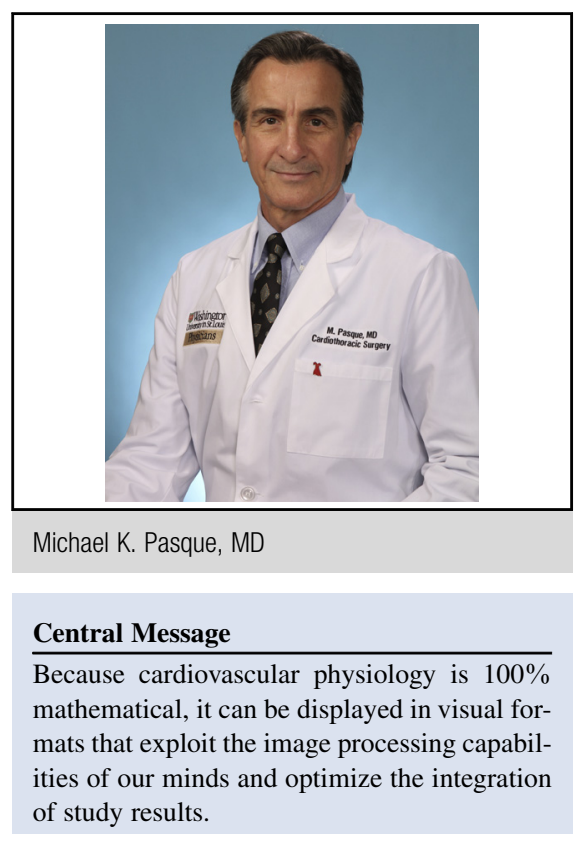

See Article page 418 . which comes already loaded into the powerful microprocessor inherent to our visual cortex, to examine critically the results of their science - and of their artistry. That which our minds have always done best is to process the trillions of tiny bits of visual information that hit our retinas every day. We process everything our gaze falls on instantaneously, automatically, in real time, and in 4 dimensions. What a tragedy that as physicians and surgeons we have been so very limited by the printed word in its meager efforts to describe that which has always been indescribable. Through the visionary leadership of this Journal, however, we find ourselves in a multidimensional, visual imagery-based paradigm shift in the reporting, dissemination, and integration of new medical knowledge.

Further, we are on the verge of a revolutionary expansion of our image analysis capabilities. Although our visual cortex performs admirably in summing massive quantities of visual data, it struggles to search out the intense relational connectivity that exists between the trillions of mathematical data points that generate the flow vectors in these images and videos. What we do know, however, is that if it can be visualized on a magnetic resonance image, somewhere it is already described mathematically. Thus in that 
very same tsunami flood of visual data is made available unimaginable information in a computationally optimized analysis format. A new generation of pattern recognition software that uses such advanced mathematical tools as support vector machines and hyperplanes can find patterns of vector association that remain unrecognized by our minds. These new pattern recognition engines can mine the countless binary signals that form the basic language of these images for the millions of relational nuances that exist between their vividly demonstrated streamlines and particle paths. These relationships, these patterns hold the deep secrets of our cardiovascular physiology. The computer has opened all of this not just to the realm of possibility but also to completion in the near future.

The only problem with the work of Oechtering and colleagues $^{2}$ is that they leave us wanting more. The application of their 4-dimensional flow magnetic resonance imaging techniques to a missing subset of patients, those who had previously undergone valve sparing root replacement with a straight, unshaped Dacron graft, would have completed the puzzle. We know that the sinus grafts supply "nearphysiologic hemodynamics," but we are also told that the patients with the sinus prosthesis had sinus vortices that were primarily "intermediate or large" relative to the "small- and intermediate-sized vortices in young and agematched volunteers." With comparable data from the straight-graft patients, we could know how much better the shaped grafts are, because we would know the physiologic range of the metric by which they are being judged. Maybe smaller sinuses would be even better!
The mathematical modeling of human cardiovascular physiology has reached critical mass. In the combination of 2 mathematical tools, computational fluid dynamics (as employed by Oechtering and colleagues ${ }^{2}$ ) and finiteelement analysis, we have everything that we need to model mathematically the whole of human cardiovascular physiology. Within this capability, the future is clear: all manmade devices that will be inserted into or substituted for any part of our cardiovascular anatomy-and therefore into our delicate balance of material properties, geometry, loading conditions, and kinetics-will be mathematically analyzed. All geometry, all movement, all blood flow will be modeled, the relationships between the various constituents explored, and behavior predicted. Within the scope of these methodologies is the ability to model mathematically and analyze computationally $100 \%$ of that which we, as cardiac surgeons, deal with every day. And most importantly, anything that can be mathematically described can also be visually displayed in 4 vivid dimensions-both before and after we apply it to our work in the operating room. This is the future of experimental biology as it pertains to our subspecialty, and we are treated to a glimpse of it in this issue of The Journal.

\section{References}

1. Galilei G. The assayer. In: Drake S translator, ed. Discoveries and opinions of Gal ileo. New York: Doubleday; 1957:237-8.

2. Oechtering TH, Hons CF, Sieren M, Hunold P, Hennemuth A, Huellebrand M et al. Four-dimensional flow magnetic resonance imaging analysis of hemodynamics in valve-sparing aortic root repair with an anatomically shaped sinus prosthesis. J Thorac Cardiovasc Surg. 2016;152:418-27.e1. 